\title{
A TeV-scale model for neutrino mass, DM and baryon asymmetry
}

\section{Mayumi Aoki}

Department of Physics, Tohoku University, Aramaki, Aoba, Sendai, Miyagi 980-8578, JAPAN

E-mail: mayumi@tuhep.phys.tohoku-u.ac.jp

\section{Shinya Kanemura*}

Department of Physics, Univeristy of Toyama, 3190 Gofuku, Toyama 930-8555, JAPAN

E-mail: kanemuesci.u-toyama.ac.jp

\section{Osamu Seto}

Instituto de Física Teórica UAM/CSIC, Universidad Autónoma de Madrid, Cantoblanco, Madrid 28049, Spain

E-mail: os amu. setoduam.es

We discuss a model which would explain neutrino oscillation, dark matter, and baryon asymmetry of the Universe simultaneously by the physics at $\mathrm{TeV}$ scales. Tiny neutrino masses are generated at the three loop level due to the exact $Z_{2}$ symmetry, by which the stability of the dark matter candidate is also guaranteed. The extra Higgs doublet is required not only for the tiny neutrino masses but also for successful electroweak baryogenesis. The model provides various discriminative predictions especially in charged Higgs phenomenology.

Prospects for Charged Higgs Discovery at Colliders

September 16-192008

Uppsala, Sweden

${ }^{*}$ Speaker. 


\section{Introduction}

Today, we have clear motivations to consider a model beyond the standard model (SM). The data indicate that neutrinos have tiny masses and mix with each other[1]. Cosmological observations have revealed that the energy density of dark matter (DM) in the Universe dominates that of baryonic matter[2]. Asymmetry of matter and anti-matter in our Universe has been addressed for several decades[3].

In this talk, we discuss a model which would explain neutrino oscillation, origin of DM and baryon asymmetry simultaneously by an extended Higgs sector with TeV-scale right-handed (RH) neutrinos[4]. Tiny neutrino masses are generated at the three loop level due to an exact discrete symmetry, by which tree-level Yukawa couplings of neutrinos are prohibited. The lightest neutral odd state under the discrete symmetry is a candidate of DM. Baryon asymmetry can be generated at the electroweak phase transition (EWPT) by additional CP violating phases in the Higgs sector[5]. In this framework, a successful model can be built without contradiction of the current data.

Original idea of generating tiny neutrino masses via the radiative effect has been proposed by Zee[6]. The extension with a TeV-scale RH neutrino has been discussed in Ref. [7], where neutrino masses are generated at the three-loop level due to the exact $Z_{2}$ parity, and the $Z_{2}$-odd $\mathrm{RH}$ neutrino is a candidate of DM. This has been extended with two RH neutrinos to describe the neutrino data[8]. Several models with adding baryogenesis have been considered in Ref. [9]. The following advantages would be in the present model: (a) all mass scales are at most at the $\mathrm{TeV}$ scale without large hierarchy, (b) physics for generating neutrino masses is connected with that for DM and baryogenesis, (c) the model parameters are strongly constrained by the current data, so that the model provides discriminative predictions which can be tested at future experiments.

\section{Model}

We introduce two scalar isospin doublets with hypercharge $1 / 2\left(\Phi_{1}\right.$ and $\left.\Phi_{2}\right)$, charged singlets $\left(S^{ \pm}\right)$, a real scalar singlet $(\eta)$ and two generation isospin-singlet RH neutrinos $\left(N_{R}^{\alpha}\right.$ with $\left.\alpha=1,2\right)$. As in Ref. [7] we impose an exact $Z_{2}$ symmetry to generate tiny neutrino masses at the three-loop level, which we refer as $Z_{2}$. We assign $Z_{2}$-odd charge to $S^{ \pm}, \eta$ and $N_{R}^{\alpha}$, while ordinary gauge fields, quarks and leptons and Higgs doublets are $Z_{2}$ even. Introduction of two Higgs doublets would cause a dangerous flavor changing neutral current. To avoid this in a natural way, we impose another discrete symmetry $\left(\tilde{Z}_{2}\right)$ that is softly broken[10]. From a phenomenological reason discussed later, we assign $\tilde{Z}_{2}$ charges such that only $\Phi_{1}$ couples to leptons whereas $\Phi_{2}$ does to quarks;

$$
\mathscr{L}_{Y}=-y_{e_{i}} \bar{L}^{i} \Phi_{1} e_{R}^{i}-y_{u_{i}} \bar{Q}^{i} \tilde{\Phi}_{2} u_{R}^{i}-y_{d_{i}} \bar{Q}^{i} \Phi_{2} d_{R}^{i}+\text { h.c. }
$$

where $Q^{i}\left(L^{i}\right)$ is the ordinary $i$-th generation left-handed (LH) quark (lepton) doublet, and $u_{R}^{i}$ and $d_{R}^{i}\left(e_{R}^{i}\right)$ are RH-singlet up- and down-type quarks (charged leptons), respectively. We summarize the particle properties under $Z_{2}$ and $\tilde{Z}_{2}$ in Table 1. Notice that the Yukawa coupling in Eq. (2.1) is different from that in the minimal supersymmetric SM (MSSM)[11]. In addition to the usual potenital of the two Higgs doublet model (THDM) with the $\tilde{Z}_{2}$ parity and that of the $Z_{2}$-odd scalars, 


\begin{tabular}{c|ccccc|cc|ccc}
\hline & $Q^{i}$ & $u_{R}^{i}$ & $d_{R}^{i}$ & $L^{i}$ & $e_{R}^{i}$ & $\Phi_{1}$ & $\Phi_{2}$ & $S^{ \pm}$ & $\eta$ & $N_{R}^{\alpha}$ \\
\hline$Z_{2}$ (exact) & + & + & + & + & + & + & + & - & - & - \\
\hline$\tilde{Z}_{2}$ (softly broken) & + & - & - & + & + & + & - & + & - & + \\
\hline
\end{tabular}

Table 1: Particle properties under the discrete symmetries.

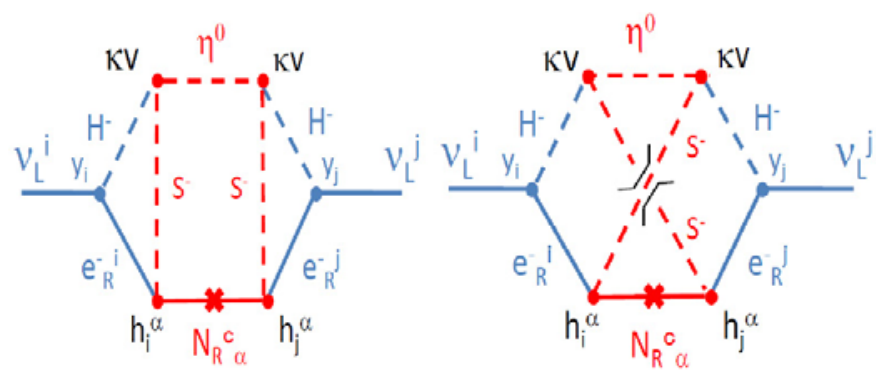

Figure 1: The diagrams for generating tiny neutrino masses.

we have the interaction terms between $Z_{2}$-even and -odd scalars:

$$
\mathscr{L}_{\text {int }}=-\sum_{a=1}^{2}\left(\rho_{a}\left|\Phi_{a}\right|^{2}|S|^{2}+\sigma_{a}\left|\Phi_{a}\right|^{2} \frac{\eta^{2}}{2}\right)-\sum_{a, b=1}^{2}\left\{\kappa \varepsilon_{a b}\left(\Phi_{a}^{c}\right)^{\dagger} \Phi_{b} S^{-} \eta+\text { h.c. }\right\},
$$

where $\varepsilon_{a b}$ is the anti-symmetric tensor with $\varepsilon_{12}=1$. The mass term and the interaction for $N_{R}^{\alpha}$ are given by

$$
\mathscr{L}_{Y_{N}}=\sum_{\alpha=1}^{2}\left\{\frac{1}{2} m_{N_{R}^{\alpha}} \overline{N_{R}^{\alpha c}} N_{R}^{\alpha}-h_{i}^{\alpha} \overline{\left(e_{R}^{i}\right)^{c}} N_{R}^{\alpha} S^{-}+\text {h.c. }\right\}
$$

Although the $\mathrm{CP}$ violating phase in the Lagrangian is crucial for successful baryogenesis at the EWPT[5], it does not much affect the following discussions. Thus, we neglect it for simplicity. We later give a comment on the case with the non-zero $\mathrm{CP}$-violating phase.

As $Z_{2}$ is exact, the even and odd fields cannot mix. Mass matrices for the $Z_{2}$ even scalars are diagonalized as in the usual THDM by the mixing angles $\alpha$ and $\beta$, where $\alpha$ diagonalizes the CPeven states, and $\tan \beta=\left\langle\Phi_{2}^{0}\right\rangle /\left\langle\Phi_{1}^{0}\right\rangle[11]$. The $Z_{2}$ even physical states are two CP-even $(h$ and $H$ ), a CP-odd $(A)$ and charged $\left(H^{ \pm}\right)$states. We here define $h$ and $H$ such that $h$ is always the SM-like Higgs boson when $\sin (\beta-\alpha)=1$.

\section{Neutrino Mass, Dark Matter, and Strongly 1st-Order Phase Transition}

The LH neutrino mass matrix $M_{i j}$ is generated by the three-loop diagrams in Fig. 1. The absence of lower order loop contributions is guaranteed by $Z_{2} . H^{ \pm}$and $e_{R}^{i}$ play a crucial role to connect LH neutrinos with the one-loop sub-diagram by the $Z_{2}$-odd states. We obtain

$$
M_{i j}=\sum_{\alpha=1}^{2} C_{i j}^{\alpha} F\left(m_{H^{ \pm}}, m_{S^{ \pm}}, m_{N_{R}^{\alpha}}, m_{\eta}\right)
$$




\begin{tabular}{|c||c|c|c|c|c|c|c|}
\hline Set & $h_{e}^{1}$ & $h_{e}^{2}$ & $h_{\mu}^{1}$ & $h_{\mu}^{2}$ & $h_{\tau}^{1}$ & $h_{\tau}^{2}$ & $B(\mu \rightarrow e \gamma)$ \\
\hline A & 2.0 & 2.0 & -0.019 & 0.042 & -0.0025 & 0.0012 & $6.9 \times 10^{-12}$ \\
\hline B & 2.2 & 2.2 & 0.0085 & 0.038 & -0.0012 & 0.0021 & $6.1 \times 10^{-12}$ \\
\hline
\end{tabular}

Table 2: Values of $h_{i}^{\alpha}$ for $m_{H^{ \pm}}\left(m_{S^{ \pm}}\right)=100(400) \mathrm{GeV} m_{\eta}=50 \mathrm{GeV}, m_{N_{R}^{1}}=m_{N_{R}^{2}}=3.0 \mathrm{TeV}$ for the normal hierarchy. For Set $\mathrm{A}(\mathrm{B}), \kappa \tan \beta=28(32)$ and $U_{e 3}=0(0.18)$. Predictions on the branching ratio of $\mu \rightarrow e \gamma$ are also shown.
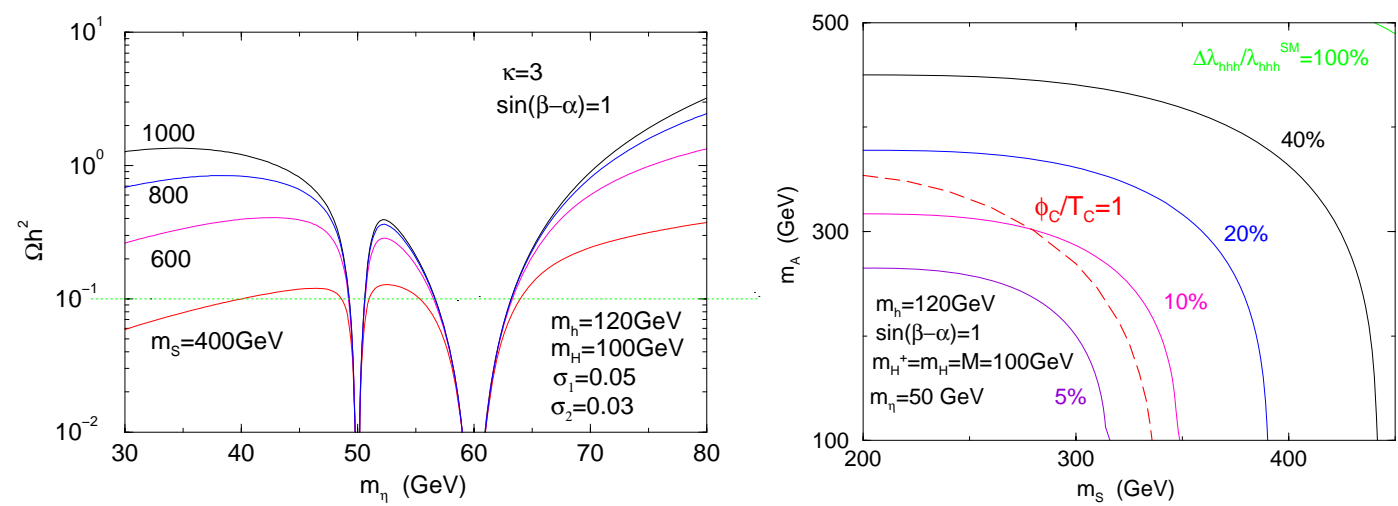

Figure 2: [Left figure] The relic abundance of $\eta$. [Right figure] The region of strong first order EWPT. Deviations from the SM value in the $h$ hh coupling are also shown.

where $C_{i j}^{\alpha}=4 \kappa^{2} \tan ^{2} \beta\left(y_{e_{i}}^{\mathrm{SM}} h_{i}^{\alpha}\right)\left(y_{e_{j}}^{\mathrm{SM}} h_{j}^{\alpha}\right)$ with $y_{e_{i}}^{\mathrm{SM}}=\sqrt{2} m_{e_{i}} / v$ and $v \simeq 246 \mathrm{GeV}$. The factor of the three-loop integral function $F\left(m_{H^{ \pm}}, m_{S^{ \pm}}, m_{N_{R}}, m_{\eta}\right)$ includes the supression factor of $1 /\left(16 \pi^{2}\right)^{3}$, whose typical size is $\mathscr{O}\left(10^{4}\right) \mathrm{eV}$. Magnitudes of $\kappa \tan \beta$ as well as $F$ determine the universal scale of $M_{i j}$, whereas variation of $h_{i}^{\alpha}(i=e, \mu, \tau)$ reproduces the mixing pattern indicated by the neutrino data[1].

Under the natural requirement $h_{e}^{\alpha} \sim \mathscr{O}(1)$, and taking the $\mu \rightarrow e \gamma$ search results into account[12], we find that $m_{N_{R}^{\alpha}} \sim \mathscr{O}(1) \mathrm{TeV}, m_{H^{ \pm}} \lesssim \mathscr{O}(100) \mathrm{GeV}, \kappa \tan \beta \gtrsim \mathscr{O}(10)$, and $m_{S^{ \pm}}$being several times $100 \mathrm{GeV}$. On the other hand, the LEP direct search results indicate $m_{H^{ \pm}}\left(\right.$and $\left.m_{S^{ \pm}}\right) \gtrsim 100 \mathrm{GeV}[1]$. In addition, with the LEP precision measurement for the $\rho$ parameter, possible values uniquely turn out to be $m_{H^{ \pm}} \simeq m_{H}$ (or $\left.m_{A}\right) \simeq 100 \mathrm{GeV}$ for $\sin (\beta-\alpha) \simeq 1$. Thanks to the Yukawa coupling in Eq. (2.1), such a light $H^{ \pm}$is not excluded by the $b \rightarrow s \gamma$ data[13]. Since we cannot avoid to include the hierarchy among $y_{i}^{\mathrm{SM}}$, we only require $h_{i}^{\alpha} y_{i} \sim \mathscr{O}\left(y_{e}\right) \sim 10^{-5}$ for values of $h_{i}^{\alpha}$. Our model turns out to prefer the normal hierarchy scenario. Several sets for $h_{i}^{\alpha}$ are shown in Table 2 with the predictions on the branching ratio of $\mu \rightarrow e \gamma$ assuming the normal hierarchy.

The lightest $Z_{2}$-odd particle is stable and can be a candidate of DM if it is neutral. In our model, $N_{R}^{\alpha}$ must be heavy, so that the DM candidate is identified as $\eta$. When $\eta$ is lighter than the $\mathrm{W}$ boson, $\eta$ dominantly annihilates into $b \bar{b}$ and $\tau^{+} \tau^{-}$via tree-level $s$-channel Higgs $(h$ and $H$ ) exchange diagrams, and into $\gamma \gamma$ via one-loop diagrams. From their summed thermal averaged annihilation rate $\langle\sigma v\rangle$, the relic mass density $\Omega_{\eta} h^{2}$ is evaluated. Fig. 2(Left) shows $\Omega_{\eta} h^{2}$ as a function of $m_{\eta}$. Strong annihilation can be seen near $50 \mathrm{GeV} \simeq m_{H} / 2\left(60 \mathrm{GeV} \simeq m_{h} / 2\right)$ due to the resonance of $H(h)$ mediation. The data $\left(\Omega_{\mathrm{DM}} h^{2} \simeq 0.11[2]\right)$ indicate that $m_{\eta}$ is around $40-65 \mathrm{GeV}$. 
The model satisfies the necessary conditions for baryogenesis[3]. Especially, departure from thermal equilibrium can be realized by the strong first order EWPT. The free energy is given at a high temperature $T$ by

$$
V_{e f f}[\varphi, T]=D\left(T^{2}-T_{0}^{2}\right) \varphi^{2}-E T \varphi^{3}+\frac{\lambda_{T}}{4} \varphi^{4}+\ldots,
$$

where $\varphi$ is the order parameter. A large value of the coefficient $E$ is crucial for the strong first order EWPT with keeping $m_{h} \lesssim 120 \mathrm{GeV}$. For sufficient sphaleron decoupling in the broken phase, it is required that[14]

$$
\frac{\varphi_{c}}{T_{c}}\left(\simeq \frac{2 E}{\lambda_{T_{c}}}\right) \gtrsim 1
$$

where $\varphi_{c}(\neq 0)$ and $T_{c}$ are the critical values of $\varphi$ and $T$ at the EWPT. In Fig. 2(Right), the allowed region under the condition of Eq. (3.3) is shown. The condition is satisfied when $m_{S^{ \pm}} \gtrsim 350 \mathrm{GeV}$ for $m_{A} \gtrsim 100 \mathrm{GeV}, m_{h} \simeq 120 \mathrm{GeV}, m_{H} \simeq m_{H^{ \pm}}(\simeq M) \simeq 100 \mathrm{GeV}$ and $\sin (\beta-\alpha) \simeq 1$.

\section{Phenomenology}

A successful scenario which can simultaneously solve the above three issues under the data[1, $12,13]$ would be

$$
\begin{array}{ll}
\sin (\beta-\alpha) \simeq 1, & (\kappa \tan \beta) \simeq 30, \\
m_{h}=120 \mathrm{GeV}, & m_{H} \simeq m_{H^{ \pm}}(\simeq M) \simeq 100 \mathrm{GeV}, \\
m_{A} \gtrsim 100 \mathrm{GeV}, \quad m_{S^{ \pm}} \sim 400 \mathrm{GeV}, \\
m_{\eta} \simeq 40-65 \mathrm{GeV}, m_{N_{R}^{1}} \simeq m_{N_{R}^{2}} \simeq 3 \mathrm{TeV} .
\end{array}
$$

This is realized without assuming unnatural hierarchy among the couplings. All the masses are between $\mathscr{O}(100) \mathrm{GeV}$ and $\mathscr{O}(1) \mathrm{TeV}$. As they are required by the data, the model has a predictive power. We note that the masses of $A$ and $H$ can be exchanged with each other.

We outline phenomenological predictions in the scenario in (4.1) in order. The detailed analysis is shown elsewhere[15]. (I) $h$ is the SM-like Higgs boson, but decays into $\eta \eta$ when $m_{\eta}<m_{h} / 2$. The branching ratio is about $36 \%(25 \%)$ for $m_{\eta} \simeq 45$ (55) GeV. This is related to the DM abundance, so that our DM scenario is testable at the LHC. (II) $\eta$ is potentially detectable by direct DM searches[16], because $\eta$ can scatter with nuclei via the scalar exchange[17]. (III) For successful baryogenesis, the $h h h$ coupling has to deviate from the SM value by more than 10-20\%[18] (see Fig. 2), which can be tested at the International Linear Collider (ILC)[19]. (IV) $H$ (or $A$ ) can predominantly decay into $\tau^{+} \tau^{-}$instead of $b \bar{b}$ for $\tan \beta \gtrsim 3$. When $A$ (or $H$ ) is relatively heavy it can decay into $H^{ \pm} W^{\mp}$ and $H Z$ (or $A Z$ ). (V) the scenario with light $H^{ \pm}$and $H$ (or $A$ ) can be directly tested at the LHC via $p p \rightarrow W^{*} \rightarrow H H^{ \pm}$and $A H^{ \pm}[20]$. (VI) $S^{ \pm}$can be produced in pair at the LHC (the ILC)[21], and decay into $\tau^{ \pm} v \eta$. The signal would be a hard hadron pair[22] with a large missing energy. (VII) The couplings $h_{i}^{\alpha}$ cause lepton flavor violation such as $\mu \rightarrow e \gamma$ which would provide information on $m_{N_{R}^{\alpha}}$ at future experiments.

Finally, we comment on the case with the CP violating phases. Our model includes the THDM, so that the same discussion can be applied in evaluation of baryon number at the EWPT[5]. The mass spectrum would be changed to some extent, but most of the features discussed above should be conserved with a little modification. 


\section{Summary}

We have discussed the model with the extended Higgs sector and TeV-scale RH neutrinos, which would explain neutrino mass and mixing, DM and baryon asymmetry by the $\mathrm{TeV}$ scale physics without less fine tuning. It gives specific predictions on many low energy phenomena. In particular, charged Higgs phenomenology in this model is completely different from that in the MSSM, so that it is testable at the LHC and the ILC.

\section{References}

[1] W. M. Yao, et al., [Particle Data Group] J. Phys. G 33 (2006) 1.

[2] E. Komatsu, et al., (WMAP Collaboration), arXiv:0803.0547 [astro-ph].

[3] A. D. Sakharov, Pisma Zh. Eksp. Teor. Fiz. 5, 32 (1967).

[4] M. Aoki, S. Kanemura and O. Seto, arXiv:0807.0361 [hep-ph].

[5] J. M. Cline, K. Kainulainen and A. P. Vischer, Phys. Rev. D 54, 2451 (1996); L. Fromme, S. J. Huber and M. Seniuch, JHEP 0611, 038 (2006).

[6] A. Zee, Phys. Lett. B 93, 389 (1980) [Erratum-ibid. B 95, 461 (1980)]; A. Zee, Phys. Lett. B 161, 141 (1985).

[7] L. M. Krauss, S. Nasri and M. Trodden, Phys. Rev. D 67, 085002 (2003).

[8] K. Cheung and O. Seto, Phys. Rev. D 69, 113009 (2004).

[9] E. Ma, Phys. Rev. D 73, 077301 (2006); J. Kubo, E. Ma and D. Suematsu, Phys. Lett. B 642, 18 (2006); T. Hambye, K. Kannike, E. Ma and M. Raidal, Phys. Rev. D 75, 095003 (2007); K. S. Babu and E. Ma, arXiv:0708.3790 [hep-ph]; N. Sahu and U. Sarkar, arXiv:0804.2072 [hep-ph].

[10] S. L. Glashow and S. Weinberg, Phys. Rev. D 15, 1958 (1977); V. D. Barger, J. L. Hewett and R. J. N. Phillips, Phys. Rev. D 41, 3421 (1990).

[11] J. F. Gunion, et al., "The Higgs Hunters's Guide” (Addison Wesley, 1990).

[12] A. Baldini, Nucl. Phys. Proc. Suppl. 168, 334 (2007).

[13] E. Barberio, et al., [Heavy Flavor Averaging Group (HFAG)], arXiv:hep-ex/0603003.

[14] G. D. Moore, Phys. Lett. B 439, 357 (1998); Phys. Rev. D 59, 014503 (1998).

[15] M. Aoki, S. Kanemura and O. Seto, in preparation.

[16] Y. D. Kim, Phys. Atom. Nucl. 69, 1970 (2006); D. S. Akerib, et al., [CDMS Collaboration], Phys. Rev. Lett. 96, 011302 (2006).

[17] J. McDonald, Phys. Rev. D 50, 3637 (1994); for a recent study, see e.g., H. Sung Cheon, S. K. Kang and C. S. Kim, J. Cosmol. Astropart. Phys. 05 (2008) 004.

[18] S. Kanemura, Y. Okada and E. Senaha, Phys. Lett. B 606, 361 (2005).

[19] M. Battaglia, E. Boos and W. M. Yao, arXiv:hep-ph/0111276; Y. Yasui, et al., arXiv:hep-ph/0211047.

[20] S. Kanemura and C. P. Yuan, Phys. Lett. B 530, 188 (2002); Q. H. Cao, S. Kanemura and C. P. Yuan, Phys. Rev. D 69, 075008 (2004).

[21] S. Kanemura, T. Kasai, G. L. Lin, Y. Okada, J. J. Tseng and C. P. Yuan, Phys. Rev. D 64, 053007 (2001).

[22] B. K. Bullock, K. Hagiwara and A. D. Martin, Phys. Rev. Lett. 67, 3055 (1991). 